\title{
An Approach for Uncertainty Aggregation using Generalised Conjunction/Disjunction Aggregators
}

\author{
Robin De Mol $^{1}$ Ana Tapia-Rosero ${ }^{1,2}$ Guy De Tré ${ }^{1}$ \\ ${ }^{1}$ Dept. of Telecommunications and Information Processing \\ Ghent University, Sint-Pietersnieuwstraat 41, B-9000 Ghent, Belgium \\ ${ }^{2}$ Dept. of Electrical and Computer Engineering \\ ESPOL University, Campus Gustavo Galindo V., Guayaquil, Ecuador
}

\begin{abstract}
Decision Support Systems are often used in the area of system evaluation. The quality of the output of such a system is only as good as the quality of the data that is used as input. Uncertainty on data, if not taken into account, can lead to evaluation results that are not representative. In this paper, we propose a technique to extend Generalised Conjunction/Disjunction aggregators to deal with uncertainty in Decision Support Systems. We first define the logic properties of uncertainty aggregation through reasoning on strict aggregators and afterwards extend this logic to partial aggregators.
\end{abstract}

Keywords: DSS, GCD, Uncertainty, Aggregation

\section{Introduction}

Decision support systems (DSS) are software tools that are generally employed to support organizational decision making activities. DSS are typically applied to system evaluation problems with a lot of systems in order to simplify the task of finding the best one [1]. Generally, a DSS computes a score for each system independently. The results can be ordered into an easy to interpret list of all evaluated systems for the decision maker (DM).

In the field of system evaluation, each system is uniquely identified by the values of its defined set of attributes. The DSS allows DMs to model evaluation criteria in the form of requirements on these attributes to define which properties describe a preferable system. The DSS uses these criteria to calculate a set of elementary preferences for each system. Using a logic interpretation, an elementary preference is the degree of truth of the statement asserting that that requirement is completely satisfied. An evaluation criterion is a compound model that uses elementary preferences to compute the global preference reflecting the global satisfaction of all requirements. The global preference is interpreted as the degree of truth of the statement that a complex system completely satisfies all requirements. Finally, all systems are ranked based on their global preference and presented to the DM.
The implementation of the evaluation criterion differentiates the different implementations of DSS [2]. In Logic Scoring of Preferences (LSP), a popular and flexible soft-computing method for system evaluation [3], this is done by means of Generalised Conjunction/Disjunction (GCD) aggregators. These allow a hierarchical construction of conditional statements with a lot of configureability, which are praised for their capability to accurately model complex decision logic.

The remainder of the paper is structured as follows. First, we discuss why uncertainty is a problem when not taken into account. Then, we explain the foundation our research is built on, the Generalised Conjunction/Disjunction. Afterwards, we introduce a strategy to deal with uncertainty, including aggregation strategies to combine uncertainty on attributes to a degree of uncertainty on the system's overall global preference. Finally, we summarize our findings in a conclusion.

\section{Problem Statement}

The quality of output generated by DSS largely depends on the quality of the analysed data, but uncertainty on attribute values from systems is often not taken into account. This could lead us to unrepresentative results hence wrong decisions being suggested to the DM.

Uncertainty can stem from different sources. For example, at the time of considering a system, certain attribute values could not be properly measured due to imperfect conditions or inaccurate tools. It can occur that a system has no data for an attribute, because it was never measured to begin with. It is also possible that a certain system has no measured data at all and obtained its values from interpolation between other systems. Additionally, sometimes attribute values are biased by the person conducting the measurement.

Uncertainty is a broad term that can be interpreted probabilistically (variance, standard deviation, interpolation) and possibilistically (vagueness, impreciseness) [4, 5]. Because of this, DSS are sometimes accused of pointing out "false positives", 
whereas the problem lies in the fact that uncertainty on the input data was ignored. It is clear that a concise technique to deal with uncertain data would prove to be a significant improvement to current DSS.

\section{Related work}

Pedrycz, Ekel and Parreiras suggest a generalized approach of multicriteria decision making under uncertainty $[5,6,7]$ based on a classic approach for monocriteria decision problems built on payoffmatrices [8] where the underlying analysis is carried out for the given solution alternatives or strategies for certain combinations of initial data or states of nature. In this technique, the idea is to compose a matrix that evaluates all possible states the systems can be in, called the payoff matrix. Then, for each system, the most optimistic and most pessimistic estimates are computed by comparing all possible outcomes for each solution alternative. Based on these, a risk can be calculated for each combination of strategy and state of nature, which indicates the difference between using that specific strategy compared to the optimal strategy for the specified certain state of nature. The resulting risk matrix then depicts how risk-inherent combinations of certain strategies and states of nature are. This technique suffers from some drawbacks, however. First, it relies on the existence and definition of possible states of nature of a system in combination with several solution strategies that are to be compared. Second, it produces an output matrix per system, making interpretation a tedious job.

Novikova and Pospelova also propose a method for multicriteria decision making under uncertainty [9], based on the minimax approach of Danskin and Germeier, for investigating outer uncertainty, which happens due to uncontrolled parameters and is assumed has a non-random nature. In their research, they make the distinction between uncertainty that is known beforehand and uncertainty that is not known. This leads to a two-step evaluation process which leads to a vector maximinimax problem. However, their solution does not mention degrees of simultaneity or replaceability or any reference to a decision support system in general, so applicability in the area we are investigating is small.

\section{Generalised Conjunction/Disjunction}

As defined by Dujmović and Larsen [1, 10], the Generalised Conjunction/Disjunction is a parametrized continuous function of at least two inputs which combines conjunctive and disjunctive properties. Through calibrating the parameter different degrees of simultaneity (andness, $\alpha$ ) and replaceability (orness, $\omega$ ) can be achieved [11]. Simultaneity and replaceability are two fundamental logic connectives that are building blocks for many decision models.
Mathematical models of simultaneity and replaceability are fundamental components of all system evaluation models. Assuming that input variables reflect the level of satisfaction of some criteria, the simultaneity is a requirement for the coincident high level of satisfaction of input criteria. All mathematical models of simultaneity reward the concurrence of high inputs, and penalize the lack of simultaneity. The most frequently used models of simultaneity are the logic functions of partial and full conjunction.

Replaceability is the dual of simultaneity. It is used in cases where any input can compensate insufficient satisfaction of other inputs. All mathematical models of replaceability penalize cases where inputs are all relatively low, and reward cases where at least one of them is sufficiently high. Typically, replaceability is modelled through the logic functions of partial and full disjunction.

The GCD comprises five degrees: full conjunction $(\wedge)$, partial conjunction $(\triangle)$, arithmetic mean, partial disjunction $(\nabla)$ and full disjunction $(\vee)$. There are several implementations of the GCD [1].

One of them is the Weighted Power Means (WPM) implementation, as defined in Figure 2, where the exponent $r$ serves as the parameter of simultaneity/replaceability. This model also introduces the use of weights assigned to each input. Choo, Schoner and Wedley have performed a study on the different ways these weights can be interpreted [12]. In our research, we interpret weights to signify the relative importance of their respective inputs but further do not investigate their contribution. To that end, the reader may assume all inputs are given an equal weight.

Dujmović, De Tré and Van de Weghe $[13,14]$ have defined 17 levels of simultaneity/replaceability ranging from the full conjunction to the full disjunction. Applied to the WPM model, this translates into 17 different values of $r$, which are shown in Table 1. This table also gives an example of the behaviour of GCD aggregators when implemented through WPM.

For inputs 1.0 and 0.0 , we can see the degree of the partial disjunction is "hard" up to the medium weak conjunction. We say any requirements combined by those aggregators are mandatory. Other variants of the partial conjunction (the weak partial conjunction and the very weak partial conjunction) are considered to combine inputs in a non-mandatory way, while still rewarding situations where all inputs are high, but all of them being high is no longer required yet still heavily penalized.

Furthermore, we can see that there is a smooth gradient of the aggregated global preference that follows the trend of $r$. The more we lean towards the full disjunction, the more orness plays a role and the more the global preference increases up to the highest degree of satisfaction among the inputs, which is finally reached at the full, pure disjunction. 


$$
y=x_{1} \diamond \ldots \diamond x_{m}= \begin{cases}x_{1} \vee \ldots \vee x_{m}, & \alpha=0, \omega=1 \\ x_{1} \nabla \ldots \nabla x_{m}, & 0<\alpha<0.5,0.5<\omega<1 \\ \left(x_{1}+\ldots+x_{m}\right) / m, & \alpha=\omega=0.5 \\ x_{1} \triangle \ldots \triangle x_{m}, & 0.5<\alpha<1,0<\omega<0.5 \\ x_{1} \wedge \ldots \wedge x_{m}, & \alpha=1, \omega=0\end{cases}
$$

Figure 1: All cases of the GCD, where $\diamond$ is a degree of andness or orness.

$$
y=F\left(x_{1}, \ldots, x_{m} ; r\right)= \begin{cases}\left(\sum_{i=1}^{m} W_{i} x_{i}^{r}\right)^{1 / r}, & 0<|r|<+\infty \\ \prod_{i=1}^{m} x_{i}^{W_{i}}, & r=0 \\ x_{1} \wedge \wedge \wedge x_{m}, & r=-\infty \\ x_{1} \vee \ldots \vee x_{m}, & r=+\infty\end{cases}
$$

Figure 2: The simplest implementation of the GCD function, where $F$ is the Weighted Power Means.

\section{Extended Generalised Conjunction/Disjunction}

In this section, we propose a strategy to incorporate uncertainty in the evaluation criterion model by extending GCD aggregators. Traditionally, a GCD aggregator is defined as a function of the form $F:[0,1]^{n} \rightarrow[0,1]$. Our extension, Extended GCD (EGCD), is defined as a vector function $U:([0,1],[0,1])^{n} \rightarrow([0,1],[0,1])$, where the abscissa of an input maps to the original preference parameter of GCD and the ordinate corresponds to a degree of uncertainty. During our research, we paid attention to the fact the global preference calculations are unchanged. Hence, in case there is no uncertainty on the inputs, the proposed technique is identical to GCD. As such, GCD can be considered as a subset of EGCD.

Note that the output of an EGCD function is again a vector. This implies the results of the system evaluation problem can no longer trivially be ranked from "best" to "worst" solution. Similarly to the cost/preference model [15], based on costbenefit analysis methods, we have deliberately chosen to keep the uncertainty parameter as a separate output parameter rather than combining it with the global preference score. Both logically and practically this makes sense: a single output would no longer allow a DM to differentiate between a system with high uncertainty yet low preference and vice versa. Furthermore, the output uncertainty can be seen as a measure of quality of the output preference and as such has meaning on its own. Finally, this also allows the DM to make a preference/uncertainty analysis after evaluating all systems, where the decision can be made to assign more or less importance to the uncertainty on a system's preference, based on the nature of the context of the problem.

\subsection{Expected behaviour}

As elementary uncertainty expresses any form of doubt that exists on its corresponding input's el- ementary preference, it follows that the aggregated global uncertainty expresses doubt on the system's global preference. As such, global uncertainty can be interpreted as a degree of how much doubt that exists on how representative the global preference of a system is how well that system satisfies the evaluation criterion.

To guard that uncertainty can not be lost or created through aggregation we define the concept of preservation of uncertainty. Mathematically this translates to a property (called "internality") that is also true for preference aggregation $[1,16]$, namely that the global uncertainty can not drop below the lowest elementary uncertainty nor can it rise above the highest elementary uncertainty.

Furthermore, the calculation of the global uncertainty output of an EGCD aggregator should not only depend on the initial elementary uncertainties of the inputs, but also on their elementary preferences.

In what follows, we have partitioned GCD aggregators into two categories: strict and partial. Among the strict aggregators, we distinguish three special cases: the full conjunction, the arithmetic mean and the full disjunction. We study these first as they are intuitive and easy to reason about. The partial aggregators form the gradient between the strict cases and are more conceptual. We hence investigate their properties afterwards by extending our logic on strict aggregators.

\subsection{On strict aggregators}

Considering the full, pure disjunction and full, pure conjunction as aggregators that select a certain input, we expect them to propagate that input's uncertainty to the output. As such, a pure aggregator's output equals its dominant (i.e., highest for disjunction and lowest for conjunction) input, for both preference and uncertainty.

However, if multiple (or all) inputs have an equal degree of elementary preference, the selection of the dominant input is no longer trivial. In that case, we first determine the set of inputs that are equally 
dominant (all inputs with the same elementary preference) before applying a tiebreaker to select the best one. In case of a disjunction, this set contains all inputs with the highest elementary preference. Dually, in case of a conjunction, the set contains all inputs with the lowest elementary preference. To select the dominant input from that set with respect to the aggregator, we use elementary uncertainties. In case of a disjunction, we are free to choose the input with the lowest uncertainty, whereas in case of a conjunction, we are forced to use the input with the highest uncertainty. As such, it can be seen that finding the dominant input in such a set follows the inverse aggregation rules of the aggregator (lowest uncertainty for disjunction, highest uncertainty for conjunction).

The arithmetic mean, on the other hand, is a weighted combination of all inputs. As such, the aggregated uncertainty of the average aggregator should also combine all elementary uncertainties in a similar fashion. Hence, we define the global uncertainty of the average aggregator as the arithmetic mean of the elementary uncertainties, with the same weights that are used to calculate the global preference.

\subsection{On partial aggregators}

We can extend this reasoning to the area of partial aggregators. Partial aggregators, unlike strict aggregators, do not simply select an input but rather produce a weighted combination of the inputs (regardless of their implementation model). In the case of WPM, the exponent $r$ of the aggregator determines which side of the spectrum the output leans to and hence which inputs are considered more "dominant" than others. As such, we propose to also calculate the global uncertainty through a weighted combination of the elementary uncertainties, with weights based on the dominance of their corresponding inputs. If an input's elementary preference is similar to the global preference (both high or both low), we say that the aggregator prefers this input and assigns a high weight to that input's uncertainty. Dually, if the input and the output are dissimilar, we say the aggregator does not prefer that input and consequently assigns a low weight to that input's elemental uncertainty. This implies that for a partial disjunction, the elementary uncertainties of inputs with high elementary preferences will be preferred, whereas for a partial conjunction, the elementary uncertainties of inputs with low elementary preferences will receive higher weights.

Importantly, the partial aggregation function should respect the three special cases. They should be seen as restrictions placed on the mathematical model that implements the partial aggregation function. Furthermore, the outputs from the partial conjunction should form a smooth gradient between the full conjunction and the average. Similarly, the outputs from the partial disjunction should form a gradient between the average and the full disjunction. Mathematically, this means the partial aggregation function should be smooth.

For binary aggregators (with exactly two inputs), we can use a simple weighted average. The weight for each input is calculated as the difference between its elementary preference and the aggregated preference, and then normalised. This behaviour is illustrated in Table 2. The results show us that the global preference calculation is, as defined, unchanged by the addition of the uncertainty parameter. Furthermore, we observe the trend of the global uncertainty meets our expectations: in the case of a full conjunction, the uncertainty of the lowest input is taken, whereas in the case of a full disjunction, the uncertainty of the highest input is propagated. Additionally, the global uncertainty of the arithmetic mean aggregator also produces the average of the elementary uncertainties. In the partial area between, we observe a gradient proportional to the global preference, namely that the global uncertainty leans to the elementary uncertainty of the preferred inputs. We also observe that the uncertainty calculations respect the mandatory behaviour of the forms of the partial conjunction.

For aggregators that work on more than two inputs, such as compound aggregators [17, 18] but also for general n-ary GCD aggregators, the desired behaviour could be modelled using splines or $n$th order Bézier curves, where the special cases impose restrictions and all other inputs are used as control points. Though this is certainly interesting to investigate in the future, it is not discussed further in this paper.

\section{Example}

To demonstrate our approach we give a small, illustrative example. Imagine we are going on a holiday to another country. There are multiple travel options, such as going by car (system A), by train and public transportation in general (system B) or by aeroplane (system $\mathrm{C}$ ). We distinguish two attributes for these travel options:

- travel time to reach the destination, and

- comfort of the transportation method.

There are many other attributes but for the example it suffices to only take these two into account.

Table 3 shows the concrete specifications of the three systems. The car system shows slight uncertainty on travel time. This uncertainty stems from the possibility of running into traffic jams or being rerouted due to road works. The comfort of travelling by car is rated as "high" as it offers a high level of freedom. It is not only possible to go exactly where you want when you want, but you can also pack any luggage you want.

The train (and public transportation in general) system has a higher travel time compared to the 


\begin{tabular}{|c|c|c|c|}
\hline System & Attribute & Value & Uncert. \\
\hline \multirow{2}{*}{ Car } & Travel time & $5 \mathrm{~h}$ & 0.2 \\
& Comfort & High & 0.0 \\
\hline \multirow{2}{*}{ Train } & Travel time & $8 \mathrm{~h}$ & 0.3 \\
& Comfort & Low & 0.1 \\
\hline \multirow{2}{*}{ Aeroplane } & Travel time & $3 \mathrm{~h}$ & 0.0 \\
& Comfort & Medium & 0.2 \\
\hline
\end{tabular}

Table 3: Estimated values for the three systems from the example.

car system. This is mainly because means of public transportation are scheduled according to time tables. Furthermore it is likely to have to transfer multiple times during transit, especially for long travels, which adds waiting time to the total travel time. There is some degree of uncertainty, however, as public transportation is rarely exactly on time. On top of this, there might be unforeseen circumstances causing for extra delays. The comfort level of the train system is considered to be "low", as the hassle of carrying your luggage around from one transport to the next and the fact you are limited in what you can take with you are restricting factors to your freedom.

It should come as no surprise that the aeroplane system is the fastest of the three options. For this attributes there is no uncertainty as aeroplanes are usually very punctual. The aeroplane system is rated between the car and the train system for its comfort level as there are still limits to your freedom regarding the amount of luggage you can pack and the personal freedom during transit, however a lot of work is done for you during departure, arrival and the flight itself. There is a certain degree of uncertainty on this value, however, as there is a chance your luggage is lost or your seat in the aeroplane is unfortunately close to a source of nuisance.

Before we can begin evaluating these systems, we need to define our preferences in the form of an evaluation criterion. This criterion is a combination (or superposition thereof) of elementary criteria on the attributes. Such an elementary criterion has to accurately reflect our preferences with respect to acceptable and unacceptable values from the domain of the corresponding attribute. Because being acceptable or not is considered to be a matter of degree, a regular fuzzy set that is defined over the set of valid domain values can be used to represent a degree of preference. Hereby a membership degree of 0 means unacceptable and a degree of 1 means fully acceptable, as demonstrated in Figure 3.

We identify the following set of elementary criteria:

- C1: Travel time is preferably short.

- C2: Comfort should be as high as possible.

Mathematically, C1 translates to a membership function that prefers low values (the second type in Figure 3 ). To define which values we find acceptable

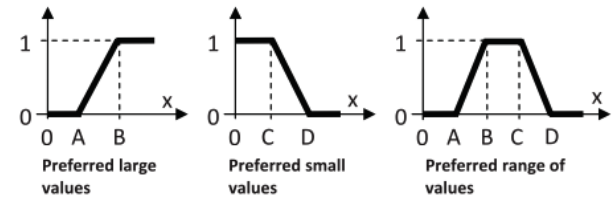

Figure 3: Membershipfunctions with linguistic interpretations.

\begin{tabular}{|c|c|c|}
\hline System & Attribute & Elem. pref. \\
\hline \multirow{2}{*}{ Car } & Travel time & 0.833 \\
& Comfort & 1.000 \\
\hline \multirow{2}{*}{ Train } & Travel time & 0.333 \\
& Comfort & 0.300 \\
\hline \multirow{2}{*}{ Aeroplane } & Travel time & 1.000 \\
& Comfort & 0.700 \\
\hline
\end{tabular}

Table 4: Computed elementary preferences for the three systems.

and unacceptable we further specify our preferences. For instance, we find that a travel time of at least 10 hours or up unacceptable yet anything below 4 hours is perfectly acceptable. For any amount of travel time between these two boundaries, we specify our preference declines linearly proportional to the amount of hours, which results in a linear downward slope between 4 and 10 hours.

C2 can be defined similarly, though here the domain of the attribute is a discrete set of values. As such, we specify a certain degree of preference for each value, where 0 denotes unacceptable and 1 indicates highly preferred. In our example, we say high comfort equals a preference of 1 , medium comfort equals a preference of 0.7 and low comfort equals a preference of 0.3 .

We use these requirements compute elementary preference scores for the travel time and comfort attributes of each system. The results of the first step of system evaluation are shown in Table 4.

In the next step, the elementary preferences with their corresponding elementary uncertainties are aggregated to a global preference and global uncertainty for each system. To combine these criteria, we use a linguistic technique proposed by Dujmović [19]. Therefore, we first need to determine if these requirements are replaceable or not. As we want them to be true simultaneously, they are not replaceable and we will compute the degree of andness to find the correct degree of partial conjunction to combine them. Then, we add a linguistic level of importance to each requirement. We say the comfort requirement is of high importance whereas the travel time requirement is only considered to be of medium importance. As such, we find the aggregator should be a Weak Conjunction [20].

The results are shown in Table 5 . From the output we learn the train system is probably the worst option as its global preference is very poor compared to the other systems. Additionally, the global un- 


\begin{tabular}{|c|c|c|}
\hline System & Preference & Uncertainty \\
\hline Car & 0.914 & 0.103 \\
\hline Train & 0.316 & 0.198 \\
\hline Aeroplane & 0.840 & 0.107 \\
\hline
\end{tabular}

Table 5: Computed global preference and uncertainty scores for three sample systems.

certainty is the highest. Given we entered expected values for the attributes and the interpretation of uncertainty is a margin for worse cases, this implies the train system's satisfaction might be more disappointing than indicated by its global preference indicator. As such, we reject the train system. The car system scores better than the aeroplane system in both preference and uncertainty, and is hence likely the best option, though both are viable alternatives.

\section{Future work}

In the future, we would like to investigate how to deal with missing data. The lacking of information can be seen as a special case of data uncertainty though we can argue to keep track of this source of uncertainty through a specific parameter to maintain a meaningful result in which it is clearly distinguishable if data is imprecise or missing. Should both be treated through the same parameter, there is no discerning two system ratings from each other, though the uncertainty might have a completely different interpretation for both systems. Though we can defend the uncertainty parameter can have multiple interpretations, it seems wise to uphold the same interpretation for each system within the same problem. Extending our research would likely result in the addition of another parameter to EGCD functions, of which the properties and the impact on preference and uncertainty aggregation need to be examined.

Furthermore, we want to investigate the possibility of extending the evaluation criterion to be more than a superposition of independent aggregator aggregators. More specifically, we want to study the feasibility of the use of contextual aggregator behaviour, where conditional executions can be controlled through an external control input.

\section{Conclusion}

In this research, we have defined a strategy for incorporating uncertainties in evaluation criteria by extending the well-known and widely used GCD aggregators. We have established a set of properties that express an intuitive logic interpretation of uncertainty aggregation. We have translated these properties into a mathematical model that an implementation of uncertainty aggregation should adhere to. For binary aggregators, we have proposed and demonstrated an implementation thereof through a weighted average function, and discussed its results. These results have shown that our model for uncertainty aggregation produces an intuitive output that is able to deal with uncertain data and produce a meaningful and interpretable output. For other aggregators, we have suggested possible implementations without further investigation, though it is clear they should also adhere to the same set of properties. We have illustrated our model through a small example to show the tangibility of our results.

\section{References}

[1] Jozo J. Dujmović and Henrik Legind Larsen. Generalized conjunction/disjunction. International Journal of Approximate Reasoning, 46(3):423-446, dec 2007.

[2] Jozo J. Dujmović, G De Tré, and S Dragicevic. Comparison of Multicriteria Methods for Landuse Suitability Assessment. IFSA/EUSFLAT Conf., 2009.

[3] Jozo J. Dujmović. Continuous preference logic for system evaluation. IEEE Transactions on Fuzzy Systems, 15(6):1082-1099, 2007.

[4] Witold Pedrycz, Petr Ekel, and Roberta Parreiras. Decision-Making in System Project, Planning, Operation, and Control: Motivation, Objectives, and Basic Concepts, pages 1-19. John Wiley \& Sons, Ltd, 2010.

[5] Witold Pedrycz, Petr Ekel, and Roberta Parreiras. Generalization of a Classic Approach to Dealing with Uncertainty of Information for Multicriteria Decision Problems, pages 247261. John Wiley \& Sons, Ltd, 2010.

[6] Petr Ya. Ekel, Illya Kokshenev, Reinaldo Palhares, Roberta Parreiras, and Fernando Schuffner Neto. Multicriteria analysis based on constructing payoff matrices and applying methods of decision making in fuzzy environment. Optimization and Engineering, 12:5-29, 2011.

[7] Petr Ya. Ekel, J.S.C. Martini, and R.M. Palhares. Multicriteria analysis in decision making under information uncertainty. Applied Mathematics and Computation, 200:501-516, 2008.

[8] Lev S Belyaev. A practical approach to choosing alternate solutions to complex optimization problems under uncertainty. 1977.

[9] Natalia M. Novikova and Irina I. Pospelova. Multicriteria decision making under uncertainty. Mathematical Programming, 92:537554, 2002.

[10] Jozo J. Dujmović. Characteristic forms of generalized conjunction/disjunction. In IEEE International Conference on Fuzzy Systems, 2008.

[11] Jozo J. Dujmović. A Comparison of Andness / Orness Indicators. In Proceedings of the 11th Information Processing and Management 
of Uncertainty international conference (IPMU 2006), page 8, San Francisco, 2006.

[12] Eng U. Choo, Bertram Schoner, and William C. Wedley. Interpretation of criteria weights in multicriteria decision making. Computers \& Industrial Engineering, 37(3):527-541, nov 1999.

[13] Jozo J. Dujmović, Guy De Tré, and Nico Van de Weghe. LSP suitability maps. Soft Computing, 14(5):421-434, June 2009.

[14] Jozo J. Dujmović and Wen Yuan Fang. Reliability of LSP criteria. In Modeling Decisions for Artificial Intelligence, volume 3131, pages 151-162. 2004.

[15] Jozo J. Dujmović, Guy De Tré, and Nico Van de Weghe. LSP suitability maps. Soft Computing, 14(5):421-434, June 2009.

[16] Jozo Dujmović. Aggregation Operators and Observable Properties of Human Reasoning. Advances in Intelligent Systems and Computing, 228:5-16, 2013.

[17] Guy De Tré, Jozo J. Dujmović, and Nico Van de Weghe. Supporting spatial decision making by means of suitability maps. Uncertainty Approaches for ..., 281, 2010.

[18] Jozo J. Dujmović and Guy De Tré. Multicriteria Methods and Logic Aggregation in Suitability Maps. International Journal of intelligent Systems, 26:971-1001, 2011.

[19] Jozo Dujmović. Andness and orness as a mean of overall importance. IEEE International Conference on Fuzzy Systems, pages 1015, 2012.

[20] Ana Tapia Rosero and Guy De Tré. Evaluating relevant opinions within a large group. In António Dourado, José Cadenas, and Joaquim Filipe, editors, 6th International Conference on Fuzzy Computation Theory and Applications, Proceedings, pages 76-86. SciTePress, 2014. 


\begin{tabular}{|l|l|c|c|}
\hline \multicolumn{1}{|c|}{ Gradient } & Symbol & Exponent r & GCD(1.0, 0.0) \\
\hline Strongest Conjunction & $\mathrm{C}$ & $-\infty$ & 0.000 \\
Very Strong Conjunction & $\mathrm{C}++$ & -9.060 & 0.000 \\
Strong Conjunction & $\mathrm{C}+$ & -3.510 & 0.000 \\
Medium Strong Conjunction & $\mathrm{C}+-$ & -1.655 & 0.000 \\
Medium Conjunction & $\mathrm{CA}$ & -0.720 & 0.000 \\
Medium Weak Conjunction & $\mathrm{C}-+$ & -0.148 & 0.000 \\
Weak Conjunction & $\mathrm{C}-$ & 0.261 & 0.070 \\
Very Weak Conjunction & $\mathrm{C}--$ & 0.619 & 0.326 \\
\hline Neutrality & $\mathrm{A}$ & 1 & 0.500 \\
\hline Very Weak Disjunction & $\mathrm{D}--$ & 1.449 & 0.620 \\
Weak Disjunction & $\mathrm{D}-$ & 2.018 & 0.709 \\
Medium Weak Disjunction & $\mathrm{D}-+$ & 2.792 & 0.780 \\
Medium Disjunction & $\mathrm{DA}$ & 3.929 & 0.838 \\
Medium Strong Disjunction & $\mathrm{D}+-$ & 5.802 & 0.887 \\
Strong Disjunction & $\mathrm{D}+$ & 9.521 & 0.930 \\
Very Strong Disjunction & $\mathrm{D}++$ & 20.63 & 0.967 \\
Strongest Disjunction & $\mathrm{D}$ & $+\infty$ & 1.000 \\
\hline
\end{tabular}

Table 1: Gradients of the partial conjunction/disjunction and an example of a GCD aggregator.

\begin{tabular}{|c|c|c|c|}
\hline $\mathbf{r}$ & $(1.0 ; 1.0),(0.0 ; 0.0)$ & $(1.0 ; 0.0),(0.0 ; 1.0)$ & $(0.2 ; 0.75),(0.7 ; 0.5)$ \\
\hline $\mathrm{C}$ & $0.000 ; 0.000$ & $0.000 ; 1.000$ & $0.200 ; 0.750$ \\
\hline $\mathrm{C}++$ & $0.000 ; 0.000$ & $0.000 ; 1.000$ & $0.216 ; 0.742$ \\
\hline $\mathrm{C}+$ & $0.000 ; 0.000$ & $0.000 ; 1.000$ & $0.243 ; 0.729$ \\
\hline $\mathrm{C}+-$ & $0.000 ; 0.000$ & $0.000 ; 1.000$ & $0.283 ; 0.708$ \\
\hline $\mathrm{CA}$ & $0.000 ; 0.000$ & $0.000 ; 1.000$ & $0.326 ; 0.687$ \\
\hline $\mathrm{C}-+$ & $0.000 ; 0.000$ & $0.000 ; 1.000$ & $0.363 ; 0.668$ \\
\hline $\mathrm{C}_{-}$ & $0.070 ; 0.070$ & $0.070 ; 0.930$ & $0.394 ; 0.653$ \\
\hline $\mathrm{C}--$ & $0.326 ; 0.326$ & $0.326 ; 0.674$ & $0.421 ; 0.639$ \\
\hline A & $0.500 ; 0.500$ & $0.500 ; 0.500$ & $0.450 ; 0.625$ \\
\hline $\mathrm{D}--$ & $0.620 ; 0.620$ & $0.620 ; 0.380$ & $0.481 ; 0.609$ \\
\hline $\mathrm{D}-$ & $0.709 ; 0.709$ & $0.709 ; 0.291$ & $0.516 ; 0.592$ \\
\hline $\mathrm{D}-+$ & $0.780 ; 0.780$ & $0.780 ; 0.220$ & $0.552 ; 0.574$ \\
\hline DD & $0.838 ; 0.838$ & $0.838 ; 0.161$ & $0.588 ; 0.556$ \\
\hline $\mathrm{D}+-$ & $0.887 ; 0.887$ & $0.887 ; 0.112$ & $0.621 ; 0.539$ \\
\hline $\mathrm{D}+$ & $0.930 ; 0.930$ & $0.930 ; 0.070$ & $0.651 ; 0.525$ \\
\hline $\mathrm{D}++$ & $0.967 ; 0.967$ & $0.967 ; 0.033$ & $0.677 ; 0.512$ \\
\hline $\mathrm{D}$ & $1.000 ; 1.000$ & $1.000 ; 0.000$ & $0.700 ; 0.500$ \\
\hline
\end{tabular}

Table 2: The output of the EGCD function for several binary configurations of inputs. 